\title{
The Use of MR Perfusion Imaging in the Evaluation of Tumor Progression in Gliomas
}

\author{
Brian Snelling, M.D., Ashish H. Shah, M.D., Simon Buttrick, M.D., Ronald Benveniste, M.D., Ph.D. \\ Department of Neurological Surgery, University of Miami Miller School of Medicine, Miami, FL, USA
}

Objective : Diagnosing tumor progression and pseudoprogression remains challenging for many clinicians. Accurate recognition of these findings remains paramount given necessity of prompt treatment. However, no consensus has been reached on the optimal technique to discriminate tumor progression. We sought to investigate the role of magnetic resonance perfusion (MRP) to evaluate tumor progression in glioma patients.

Methods : An institutional retrospective review of glioma patients undergoing MRP with concurrent clinical follow up visit was performed. MRP was evaluated in its ability to predict tumor progression, defined clinically or radiographically, at concurrent clinical visit and at follow up visit. The data was then analyzed based on glioma grade and subtype.

Resusts : A total of 337 scans and associated clinical visits were reviewed from 64 patients. Sensitivity, specificity, positive and negative predictive value were reported for each tumor subtype and grade. The sensitivity and specificity for high-grade glioma were $60.8 \%$ and $87.8 \%$ respectively, compared to low-grade glioma which were $85.7 \%$ and $89.0 \%$ respectively. The value of MRP to assess future tumor progression within 90 days was $46.9 \%$ (sensitivity) and $85.0 \%$ (specificity).

Conclusion : Based on our retrospective review, we concluded that adjunct imaging modalities such as MRP are necessary to help diagnose clinical disease progression. However, there is no clear role for stand-alone surveillance MRP imaging in glioma patients especially to predict future tumor progression. It is best used as an adjunctive measure in patients in whom progression is suspected either clinically or radiographically.

Key Words : Glioma · Tumor progression · Radiation necrosis · Magnetic resonance perfusion.

\section{INTRODUCTION}

Differentiating post-treatment changes in high-grade gliomas remains a challenging clinical task due to their radiological similarities (recurrent enhancing lesions). The prompt diagnosis of tumor progression and radiation necrosis re- mains paramount due to the apparent therapeutic and prognostic implications. Accurate diagnoses with conventional MR imaging are limited, as tumor progression appears to strongly mimic entities such as radiation necrosis and pseudoprogression as outlined by the MacDonald criteria ${ }^{19)}$. Over the last decade, Response Assessment in Neuro-Oncology

- Received: February 1, 2016 • Revised: August 15, 2016 •Accepted: August 30, 2016

- Address for reprints : Ronald J. Benveniste, M.D, Ph.D.

Department of Neurological Surgery, University of Miami School of Medicine, 1095 NW Terrace Miami, FL 33136, USA

Tel : +1-305-243-6946, Fax : +1-305-243-3337, E-mail : RBenveniste@med.miami.edu

This is an Open Access article distributed under the terms of the Creative Commons Attribution Non-Commercial License (http://creativecommons.org/licenses/by-nc/4.0) which permits unrestricted non-commercial use, distribution, and reproduction in any medium, provided the original work is properly cited. 
criteria has replaced the McDonald criteria to help discriminate these entities more clearly by dividing tumor response into four sets (complete, partial, stable, and progression) ${ }^{25}$. Recently, it has been demonstrated that accurate identification of tumor progression may be enhanced by adjunct imaging modalities such as magnetic resonance perfusion (MRP), Single photon emission computed tomography (SPECT) or positron emission tomography (PET) imaging $^{3-5,7,11,12,15,24)}$.

MRP is a unique imaging modality that utilizes gadolinium contrast to expand upon conventional MRI sequencing in order to approximate the blood flow/volume of selected tissues. Neoplasms, due to their high vascularity, tend to demonstrate higher cerebral blood volume (CBV) measurements than normal brain. Radiation necrosis on the other hand tends to demonstrate lower CBV values due to the lower cellularity and lack of neovascularization ${ }^{8,16,22)}$. Because of its availability at most hospitals, MRP may be an effective tool for rapidly distinguishing these two entities.

Many patients in our institution receive surveillance MRP imaging to evaluate for glioma recurrence. In our institution, it is used in three different scenarios: First, it is used contemporaneously with MR and/or clinical findings suggestive of TP in patients with treated high grade glioma, as an adjunct to determine whether there is true TP or RN or pseudo-progression (PP). Second, it is used contemporaneously with MR and/or clinical findings suggestive of tumor progression in patients with low grade glioma, as an adjunct to determine whether there has been progression to a higher grade of tumor. Finally, it is used as a "screening tool" in patients with any type of glioma and stable clinical and imaging findings, to try to predict progression at a later date. Since there is no consensus in the literature on the utility of MRP in confirming clinical tumor progression for any of the three scenarios, we aimed to elucidate the clinical accuracy of MRP more closely by reviewing our own institutional experience.

\section{MATERIALS AND METHODS}

\section{Selection criteria}

Following approval for our institutional review board (IRB), we conducted a retrospective chart review of patients who received MRP for surveillance of TP. Patients were included who had histopathologically proven glioma ([World Health Organization, WHO] II-VI) and who received neurooncology care and surveillance MRP imaging in conjunction with their follow-up visit at our institution. Patients who received MRP imaging for diagnoses other than glioma were excluded. Patients with less than two MRP studies were also excluded. Patients with follow up clinical visits without concordant imaging, or vice versa, were also excluded.

\section{Study definitions}

$\mathrm{TP}$, based on the revised assessment in neuro-oncology (RANO) criteria, was identified by any of the following: significant increase in size of enhancing lesions on stable or increased steroid dosage, a significant increase in T2/fluid attenuated inversion recovery (FLAIR) nonenhancing lesions, appearance of new lesions, or clear clinical deterioration not due to an apparent comorbid condition. If the patient had any evidence of clinical, radiographic RANO or histological tumor progression, it was considered tumor progression as deemed by a board-certified neuro-oncologist. Histological data was routinely not available as many patents had tumor progression but were not taken to the operating room. MRP was performed by injecting contrast agent during a dynamic susceptibility contrast scan to quantify gadolinium uptake over time. The cerebral blood flow was then qualitatively analyzed using a color gradient to compare to non-pathogenic areas. MRP was defined as positive based on a significant increase in CBV as determined qualitatively by an attending neuroradiologist blinded to the clinical data; no specific threshold values were used. MRP imaging was defined as contemporaneous if it occurred within seven days, but not after the corresponding clinical visit. 


\section{Data analysis}

For contemporaneous visits, TP, defined as positive or negative by the criteria above (radiographic, histological or clinical tumor progression), was the "gold standard" and MRP, also defined above, as the "test", i.e., a positive MRP study with a concurrent determination of clinical or radiographic progression would be deemed true positive. The positive predictive value (PPV), negative predictive value (NPV), sensitivity and specificity were calculated with subgroup analysis based on WHO grade to assess the utility of MRP in predicting tumor progression as deemed by the above criteria.

Analysis of MRP in detecting TP at the next follow up clinical visit was also analyzed, with MRP at the current visit as the "test" and TP at following follow up clinical visit as the "gold standard". PPV, NPV, sensitivity, and specificity were then calculated. Clinical and radiographic encounters were excluded if the length from MRP study to next clinical follow up visits was greater than ninety days. Statistical analysis was performed by one author (AS) and confirmed by a second (BS). Pathological data was included when available as the definitive indication of true tumor progression.

\section{RESULTS}

A total of 337 MRP studies and associated contemporaneous clinical visits were reviewed from 64 patients. 27/64 (42.1\%) patients were male. Twenty-four patients had WHO II gliomas, seventeen were WHO III, and twenty-three were WHO IV. Of these patients, 20 patients had oligodendrogliomas or oligodendroglial components to their tumors, of which 14 were WHO II and 6 WHO III. 61 patients had prior surgery; 3 patients did not have adequate records of prior surgery. 50 of 59 patients underwent prior radiation, with 5 patients having insufficient documentation as to prior radiation treatment. Patient demographics are listed in Table 1.

\section{Contemporaneous clinical visits}

For contemporaneous MRP imaging studies and clinical
Table 1. Baseline characteristics of the 64 subjects

\begin{tabular}{lc}
\hline Patient characteristics & Value \\
\hline Age (years) & 47.6 \\
Gender & $27(42.1)$ \\
$\quad$ Male, n (\%) & $61(95.3)$ \\
Prior surgery & $50(84.7)$ \\
Prior radiation, n (\%) & \\
WHO & $24(37.5)$ \\
$\quad$ II, n (\%) & $17(26.5)$ \\
$\quad$ III, n (\%) & $23(35.9)$ \\
$\quad$ IV, n (\%) & $20(31.2)$ \\
\hline Oligodendroglial component, n (\%)
\end{tabular}

Values are presented as number (\%). WHO : World Health Organization

Table 2. Sensitivity and Specificity of MRP in predicting clinicoradiographic tumor progression

\begin{tabular}{lcccc}
\hline & Sensitivity & Specificity & PPV & NPV \\
\hline LGG & 85.7 & 89.9 & 76.9 & 94.1 \\
HGG & 60.8 & 87.8 & 72.6 & 80.8 \\
Predictive & 46.9 & 85.0 & 71.4 & 66.7 \\
\hline
\end{tabular}

Sensitivity, specificity, positive predictive value, and negative predictive value of MRP for LGG and HGG at a contemporaneous clinic visit, as well as at a delayed follow up visit within 90 days (row 3). MRP : magnetic resonance perfusion, LGG : low-grade gliomas, HGG : high-grade gliomas, PPV : positive predictive value, NPV : negative predictive value

visits, the total PPV, NPV, sensitivity and specificity for both LGG and HGG were calculated (Table 2) from the clinical visits and concurrent imaging (Table 3). The average number of MRP imaging studies and associated visits for each patient was 5.3. The sensitivity, specificity and positive predictive value of MRP to analyze tumor progression in LGG was $85.7 \%, 89.0 \%$, and $76.9 \%$ respectively. The sensitivity, specificity and positive predictive value of MRP to analyze HGG tumor progression was $60.8 \%, 87.8 \%$, and $72.6 \%$ respectively.

\section{Future clinical visits}

The ability of MRP to evaluate for tumor progression at the next clinical visit was also assessed. One-hundred and twenty-five MRP studies and associated follow-up clinical visits occurred within 90 days. The PPV, NPV, sensitivity and specificity for all glioma types were calculated (Table 2) from the imaging and next follow up visits less than ninety days. Pre- 
Table 3. Clinicoradiographic tumor progression and MRP association

\begin{tabular}{|c|c|c|c|c|}
\hline & $\begin{array}{l}\text { Progression } \\
\text { and MRP + }\end{array}$ & $\begin{array}{l}\text { Progression } \\
\text { and MRP - }\end{array}$ & $\begin{array}{c}\text { Stable and } \\
\text { MRP + }\end{array}$ & $\begin{array}{l}\text { Stable and } \\
\text { MRP- }\end{array}$ \\
\hline$\overline{L G G}$ & 30 & 5 & 9 & 80 \\
\hline HGG & 45 & 5 & 29 & 122 \\
\hline Predictive & 30 & 34 & 12 & 68 \\
\hline
\end{tabular}

Rows 1 and 2 summarize the number patients with MRP findings and clinical tumor progression by RANO criteria at a contemporaneous clinic visit for low-grade and high-grade gliomas, respectively. Row 3 summarizes MRP findings, matched with clinical tumor progression at a delayed follow up visit within 90 days, thus evaluating the predictive capacity of MRP in our sample. MRP+ designates positive MRP based on the neuroradiologist's review, MRP- designates negative MRP. MRP : magnetic resonance perfusion, LGG : low-grade glioma, HGG : high-grade glioma, RANO : revised assessment in neuro-oncology

dictive value of MRP to assess tumor progression within 90 days was $46.9 \%$ (sensitivity) and $85.0 \%$ (specificity).

\section{DISCUSSION}

The results from our review show that as a measure of surveillance for TP, contemporaneous MRP performed in a manner similar to other adjunct imaging modalities. MRP did not perform well in its ability to predict TP at the next clinical visit. These results demonstrate that the use of MRP is likely most valuable as an adjunctive measure in patients who are already suspected of having TP, as opposed to a routine surveillance imaging modality.

\section{MRP for high-grade gliomas}

The contemporaneous use of MRP in conjunction with MR for evaluation of tumor progression in high-grade gliomas has been well characterized in the literature $\mathrm{s}^{3,8,10,11,16,21,23)}$. The sensitivity and specificity of MRP to predict histological tumor progression in conjunction with MR has been approximately $80 \%$ and $77 \%$ respectively ${ }^{24)}$. Additionally, the utility of MRP increases if a specific cut-off parameter was used for characterizing TP. For example, Kim and Narang et al. reported near $100 \%$ diagnostic accuracy in cases when cutoff values were set for CBV (cerebral blood volume ratio) and MSIVP (maximum slope of enhancement in the vascular phase) respectively ${ }^{17,20)}$. By using MRP alone, the sensitivi- ty and specificity of detecting tumor progression in our series was approximately $61 \%$ and $88 \%$ respectively. Our series indicates that when using MRP in conjunction with both standard MR and clinical follow-up, the accuracy of MRP increases. However, in our study, specific cut-off parameters were not utilized to optimize the specificity of our study. Additionally, in comparison to other studies which only used tumor histology as evidence of progression, our study compares MRP to radiographic and clinical progression as well which is more indicative of daily decision-making for neurooncologists as many patients are not-reoperated everytime a MR imaging displayes progression.

\section{MRP for low-grade gliomas}

Low grade-gliomas typically may or may not enhance on imaging, and when enhancement is present, anaplastic (WHO Grade III) features may be present. However, after radiation, differentiation of post-treatment enhancement on MR and tumor progression for low-grade gliomas becomes challenging due to their delayed malignant transformation and potential for radiation necrosis ${ }^{13)}$. Use of perfusion imaging in these low-grade tumors may detect malignant transformation with reasonable accuracy. In our series, sensitivity and specificity remained high for these lesions using MRP. In addition, other series seem to endorse following changes in CBV for low-grade gliomas to detect anaplastic transformation as early as 1 year prior to the development of contrst-enhancement ${ }^{6,9,18)}$. However, other modalities may be more efficacious in detecting progression earlier compared to MRP. Hlaihel et al. noted that magnetic resonance spectroscopy detected tumor progression (based on increased choline/creatine ratio) on average 15 months prior to increases in CBV on $\mathrm{MRP}^{14)}$. Nevertheless, MRP with contemporaneous MR may be useful if careful cut-off parameters are utilized to detect clinical tumor progression.

\section{Use of MRP as a screening tool}

Perfusion imaging as a screening tool to predict malignant transformation in patients with any glioma remains important. In our study, perfusion imaging did not accurately pre- 
dict tumor progression at the next clinic visit, yet was functionally useful for contemporaneous evaluation of disease status. The lack of predictive value maybe linked to the fact that no specific cut-off value was employed for CBV as many studies have noted higher CBV values several months prior to tumor progression. A multi-parametric prospective study using spectroscopy and perfusion may be helpful in delineating tumor progression in glioma patients. Our study, however indicates that general increased CBV values on perfusion imaging, by itself, are not suggestive of future tumor progression. We therefore recommend combined modality screening tests including MR spectroscopy and perfusion for accurate predictions of disease status.

\section{Study limitations}

Our study was limited in several areas. First, while the criteria for TP was based on RANO criteria, significant increases in contrast enhancement, T2/FLAIR signal, and CBV were defined at the discretion of the neuroradiologist. More standardized definitions of positive MRP imaging or radiographic progression using threshold values would increase intra- and inter-observer reliability. Additionally, pathological confirmation is the accepted standard for defining true tumor progression, so our radiological association based on RANO criteria merely serves as a surrogate. Also, our mean number of days between clinical follow up was in excess of four months. Thus, only 125 of 273 follow up visits fell within 90 days. The average number of days from MRP to follow up visit, even after excluding visits greater than 90 days, was 63 days. As such, the likelihood of MRP to predict TP would have increased had the length been shorter.

Additionally, the utility of perfusion-based imaging dramatically decreases after certain types of treatment such as anti-angiogenic chemotherapeutics and brachytherapy ${ }^{1,2}$. Variations in contrast-enhancement patterns after bevacizumab treatment confounds the utility of perfusion imaging. In our study, a few recurrent tumors were treated with bevacizumab after confirmed recurrence, which may also impact the accuracy of MRP. Nevertheless, recently, studies have begun to use standardized CBV parameters and other MRI- based biomarkers to overcome these barriers to effectively monitor treatment response after bevacizumab treatment. A future prospective study to evaluate the value of these parameters in post-bevacizumab treated patients may be useful.

\section{CONCLUSION}

MRP represents a promising imaging modality in evaluating TP in glioma patients. Our study reveals that MRP performs similarly to other adjunctive diagnostic imaging studies (MRS, SPECT) in evaluating TP at contemporaneous clinic visits, but fared poorly in its ability to predict clinical or radiographic progression at the subsequent visit.

While its role has not yet been clearly defined, perhaps the use of MRP currently is best done as an adjunct with other imaging modalities, such as MRS and PET, when TP is suspected as opposed to a measure of routine stand-alone surveillance. Although our study is limited due to the lack of histological confirmation, these results remain practical for neuro-oncologists who routinely change treatment plans based on clinical/radiographic signs of progression. Further studies need to undertaken to develop standardized criteria for TP on MRP before it could be used as a surveillance measure for all glioma patients.

\section{References}

1. Aiken AH, Chang SM, Larson D, Butowski N, Cha S : Longitudinal magnetic resonance imaging features of glioblastoma multiforme treated with radiotherapy with or without brachytherapy. Int J Radiat Oncol Biol Phys 72 : 1340-1346, 2008

2. Artzi M, Bokstein F, Blumenthal DT, Aizenstein O, Liberman G, Corn BW, et al. : Differentiation between vasogenic-edema versus tumor-infiltrative area in patients with glioblastoma during bevacizumab therapy: a longitudinal MRI study. Eur J Radiol 83 : 1250-1256, 2014

3. Arvinda HR, Kesavadas C, Sarma PS, Thomas B, Radhakrishnan VV, Gupta AK, et al. : Glioma grading: sensitivity, specificity, positive and negative predictive values of diffusion and perfusion imaging. J Neurooncol $94: 87-96,2009$

4. Barajas RF Jr, Chang JS, Segal MR, Parsa AT, McDermott MW, Berger MS, et al. : Differentiation of recurrent glioblastoma multiforme from 
radiation necrosis after external beam radiation therapy with dynamic susceptibility-weighted contrast-enhanced perfusion MR imaging. Radiology 253 : 486-496, 2009

5. Bisdas S, Naegele T, Ritz R, Dimostheni A, Pfannenberg C, Reimold M, et al. : Distinguishing recurrent high-grade gliomas from radiation injury: a pilot study using dynamic contrast-enhanced MR imaging. Acad Radiol 18 : 575-583, 2011

6. Bobek-Billewicz B, Stasik-Pres G, Hebda A, Majchrzak K, Kaspera W, Jurkowski M : Anaplastic transformation of low-grade gliomas (WHO II) on magnetic resonance imaging. Folia Neuropathol 52 : 128-140, 2014

7. Bobek-Billewicz B, Stasik-Pres G, Majchrzak H, Zarudzki L : Differentiation between brain tumor recurrence and radiation injury using perfusion, diffusion-weighted imaging and MR spectroscopy. Folia Neuropathol $48: 81-92,2010$

8. Chung WJ, Kim HS, Kim N, Choi CG, Kim SJ : Recurrent glioblastoma: optimum area under the curve method derived from dynamic contrastenhanced T1-weighted perfusion MR imaging. Radiology 269 : 561568, 2013

9. Danchaivijitr N, Waldman AD, Tozer DJ, Benton CE, Brasil Caseiras G, Tofts PS, et al. : Low-grade gliomas: do changes in rCBV measurements at longitudinal perfusion-weighted MR imaging predict malignant transformation? Radiology 247 : 170-178, 2008

10. Di Costanzo A, Scarabino T, Trojsi F, Popolizio T, Bonavita S, de Cristofaro $\mathrm{M}$, et al. : Recurrent glioblastoma multiforme versus radiation injury: a multiparametric 3-T MR approach. Radiol Med 119 : 616-624, 2014

11. Fatterpekar GM, Galheigo D, Narayana A, Johnson G, Knopp E : Treatment-related change versus tumor recurrence in high-grade gliomas: a diagnostic conundrum--use of dynamic susceptibility contrast-enhanced (DSC) perfusion MRI. AJR Am J Roentgenol 198 : 19-26, 2012

12. Gomez-Rio M, Rodriguez-Fernandez A, Ramos-Font C, Lopez-Ramirez E, Llamas-Elvira JM : Diagnostic accuracy of 201Thallium-SPECT and 18FFDG-PET in the clinical assessment of glioma recurrence. Eur J Nucl Med Mol Imaging 35 : 966-975, 2008

13. Guillevin R, Herpe G, Verdier M, Guillevin C : Low-grade gliomas: The challenges of imaging. Diagn Interv Imaging 95 : 957-963, 2014

14. Hlaihel C, Guilloton L, Guyotat J, Streichenberger N, Honnorat J, Cotton $\mathrm{F}$ : Predictive value of multimodality MRI using conventional, perfusion, and spectroscopy MR in anaplastic transformation of low-grade oligodendrogliomas. J Neurooncol 97 : 73-80, 2010

15. Kahn D, Follett KA, Bushnell DL, Nathan MA, Piper JG, Madsen M, et al. : Diagnosis of recurrent brain tumor: value of 201TI SPECT vs 18F-fluorodeoxyglucose PET. AJR Am J Roentgenol 163 : 1459-1465, 1994
16. Kim HS, Goh MJ, Kim N, Choi CG, Kim SJ, Kim JH : Which combination of MR imaging modalities is best for predicting recurrent glioblastoma? Study of diagnostic accuracy and reproducibility. Radiology 273 : 831 843,2014

17. Kim YH, Oh SW, Lim YJ, Park CK, Lee SH, Kang KW, et al. : Differentiating radiation necrosis from tumor recurrence in high-grade gliomas: assessing the efficacy of 18F-FDG PET, 11C-methionine PET and perfusion MRI. Clin Neurol Neurosurg 112 : 758-765, 2010

18. Law M, Young RJ, Babb JS, Peccerelli N, Chheang S, Gruber ML, et al. : Gliomas: predicting time to progression or survival with cerebral blood volume measurements at dynamic susceptibility-weighted contrastenhanced perfusion MR imaging. Radiology 247 : 490-498, 2008

19. Macdonald DR, Cascino TL, Schold SC Jr, Cairncross JG : Response criteria for phase II studies of supratentorial malignant glioma. J Clin Oncol 8 : 1277-1280, 1990

20. Narang J, Jain R, Arbab AS, Mikkelsen T, Scarpace L, Rosenblum ML, et al. : Differentiating treatment-induced necrosis from recurrent/progressive brain tumor using nonmodel-based semiquantitative indices derived from dynamic contrast-enhanced T1-weighted MR perfusion. Neuro Oncol 13 : 1037-1046, 2011

21. Prat R, Galeano I, Lucas A, Martinez JC, Martin M, Amador R, et al. : Relative value of magnetic resonance spectroscopy, magnetic resonance perfusion, and 2-(18F) fluoro-2-deoxy-D-glucose positron emission tomography for detection of recurrence or grade increase in gliomas. J Clin Neurosci $17:$ 50-53, 2010

22. Raimbault A, Cazals X, Lauvin MA, Destrieux C, Chapet S, Cottier JP : Radionecrosis of malignant glioma and cerebral metastasis: A diagnostic challenge in MRI. Diagn Interv Imaging 95 : 985-1000, 2014

23. Seeger A, Braun C, Skardelly M, Paulsen F, Schittenhelm J, Ernemann U, et al. : Comparison of three different MR perfusion techniques and MR spectroscopy for multiparametric assessment in distinguishing recurrent high-grade gliomas from stable disease. Acad Radiol 20 : 1557-1565, 2013

24. Shah AH, Snelling B, Bregy A, Patel PR, Tememe D, Bhatia R, et al. : Discriminating radiation necrosis from tumor progression in gliomas: a systematic review what is the best imaging modality? J Neurooncol 112 : 141-152, 2013

25. Wen PY, Macdonald DR, Reardon DA, Cloughesy TF, Sorensen AG, Galanis $E$, et al. : Updated response assessment criteria for high-grade gliomas: response assessment in neuro-oncology working group. J Clin Oncol 28 : 1963-1972, 2010 